\title{
Biología de Cotesia flavipes Cameron sobre el Taladrador del Tallo, Diatraea saccharalis Fabricius, en Varios Medios de Cría
}

\section{Biology of Cotesia flavipes Cameron on Stem Borer, Diatraea saccharalis Fabricius in Various Rearing Media}

Erick Vélez ${ }^{1}$, Rossana Castro ${ }^{2}$, Dorys T. Chirinos ${ }^{3, *}$

INFORMACIÓN DEL

ARTÍCULO

Fecha de recepción: 31 de enero de 2020.

Fecha de aceptación: 19 de febrero de 2020.

${ }^{1}$ Estudiante del Postgrado. Maestría en Agronomía, Mención Producción Agrícola Sostenible. Universidad Técnica de Manabí, Código Postal 130105, Portoviejo, Manabí, Ecuador.

2 Investigadora Independiente, Guayaquil, Ecuador.

3 Facultad de Ingeniería Agronómica, Universidad Técnica de Manabí, Código Postal 130105, Portoviejo, Manabí, Ecuador.

* Autor de correspondencia: Dorys T. Chirinos, Facultad de Ingeniería Agronómica, Universidad Técnica de Manabí, Código Postal 130105, Portoviejo, Manabí, Ecuador. E-mail: dtchirinos@gmail.com Tlf.: (593-5) 263-2677.

ENLACE DOI http://dx.doi.org/10.31095/investigatio 2020.13 .8

\section{Resumen}

El barrenador del tallo, Diatraea saccharalis, es considerado el insecto plaga más relevante en caña de azúcar, un cultivo importante en Ecuador. Cotesia flavipes constituye uno de sus agentes de control biológico, para el cual, se estudiaron: aspectos biológicos, cantidad de larvas parasitadas y el efecto de la dieta, recipiente de cría y edad de la larva hospedera sobre el parasitismo. El parasitoide pasó por tres estadíos larvales, completando su ciclo en 16 días. La reproducción es arrenotoquica con una proporción de 2,11:1 (hembras: macho). Independiente del número de larvas expuestas, una hembra solo parasitó una larva del hospedero de donde emergieron aproximadamente 40 pupas. La mejor combinación para el desarrollo de los parasitoides fue el recipiente de cría perforado con larva hospedera de 18 días de edad alimentada con maíz tierno $(\mathrm{P}<0,05)$. Los resultados aportan elementos a considerar para la cría masiva de este importante agente de control biológico.

\section{Palabras Clave:}

Control biológico, crías masivas, parasitoide.

Clasificación JEL: Q100.

\section{Abstract}

The stem borer, Diatraea saccharalis, is considered to be the most relevant pest insect in sugarcane, an important crop in Ecuador. Cotesia flavipes is one of its biological control agents. Biological aspects of this parasitoid, amount of parasitized larvae and the effect of diet, breeding containers and age of the host larva on parasitism were studied. The parasitoid went through three larval stages, and completed its cycle in 16 days. Reproduction is arrhenotokous with a ratio of 2.11 females for each male. Regardless of the number of larvae exposed, a female only parasitized one larva of the host from which about 40 pupae emerged. The best combination for the development of parasitoids was the 18-day-old host perforated container fed with young corn $(\mathrm{P}<0.05)$. The results provide elements to consider for the mass breeding of this important biological control agent.

\section{Keywords:}

Biological control, mass rearing, parasitoid.

JEL Classification: Q100.

77

INVESTIGATIO No. 13, marzo 2020, pp. 77-88,

ISSN: 1390 - 6399・ISSN-e: 2602 - 8336 


\section{Introducción}

Ecuador es uno de los principales países productores de caña de azúcar, Saccharum oficinarum L. en el que se cultivan 116.483 ha con una producción de $9.030 .073 \mathrm{Tm}$, en el cual, la provincia de Guayas concentra la mayor producción con el $82,83 \%$ del total nacional (Instituto Nacional de Estadística y Censos INEC, 2017). Su importancia radica en que está considerada una de las principales fuentes de energía del país junto con el arroz, maíz y yuca (Pérez y Rodríguez, 2018).

Tal como ocurre en otros cultivos, la producción de caña de azúcar puede ser limitada por problemas fitosanitarios, entre éstos, el barrenador del tallo, Diatraea saccharalis F. (Lepidoptera: Crambidae) que es considerada la plaga más relevante de la caña en el continente americano (Vacari et al., 2010; Cordoba y Soto, 2015). Se trata de un insecto que en su estado larval taladra los tallos tanto en plantas jóvenes como adultas y en consecuencia reduce la producción y calidad de la caña (Aya et al., 2017). En la actualidad, para ejercer un control sobre la misma, es notable el uso de agentes de control biológico, lo cual implica beneficios, pues son capaces de llegar a sitios donde los insecticidas no pueden hacer contacto con el insecto objeto de tratamiento, aunado al hecho que se reduce el uso de estos factores de control químico que generalmente son de moderado a alto impacto ambiental.

Uno de los agentes de control biológico de $D$. saccharalis, lo constituye
Cotesia flavipes, Cameron (Hymenoptera: Braconidae), un parasitoide gregario con una alta perspectiva para control de este barrenador y otros barrenadores de tallos (Ferrer y Liniares, 1990; Khan et al., 2017). Los antecedentes indican que de un solo hospedero parasitado por $C$. flavipes es posible obtener de 30 a 110 adultos aproximadamente (Muirhead et al., 2010), razón por la cual puede ser producido eficientemente en masa; sin embargo, para la producción en masa son necesarios varios factores, entre los que destacan, asepsia, esterilidad, temperatura, humedad y el manejo en el laboratorio, para que no se vea afectada la producción del parasitoide (Fajardo y Mendoza, 1993).

En algunos laboratorios de cría masiva de $C$. flavipes (Guayas, Ecuador), la producción de este parasitoide se ha visto afectada porque los porcentajes de parasitismo fueron disminuyendo en el tiempo alcanzando solo un promedio de $7,5 \%$ entre los ingenios azucareros San Carlos, Escudos y Valdez (Mendoza et al., 2009). Esta disminución ha sido atribuida a las condiciones de cría, pero sin precisar los aspectos específicos que podrían estar afectando el parasitismo. Otras investigaciones refieren efectos sobre la biología del parasitoide de algunos factores, entre los que destacan, las condiciones climáticas, composición de la dieta, así como, de la edad y calidad de la larva hospedera (Eduardo et al., 2017; Khan et al., 2017). Por esta razón, esta investigación tuvo como objetivo, evaluar el efecto de algunos de los factores, como el tipo de dieta y los recipientes de cría y la edad de la larva 
hospedera que podrían incidir en la eficiencia del parasitismo, así como, el estudio de algunos aspectos biológicos de C. flavipes parasitoide de D. saccharalis.

\section{Materiales y métodos}

La presente investigación se llevó a cabo en el Laboratorio de Entomología (T: 25,2 ${ }^{\circ} \mathrm{C}$ y HR: $69 \%$ ), Facultad de Ciencias Agrarias, Universidad Agraria del Ecuador durante el período febreroagosto de 2016. Para los experimentos, los parasitoides iniciales fueron obtenidos del Laboratorio de Entomología, Sociedad Agrícola e Industrial San Carlos S.A., cantón Marcelino Maridueña, Provincia del Guayas.

Duración del ciclo biológico. Fueron expuestas 206 larvas de D. saccharalis 18 días de edad (de cuarto estadío), a hembras apareadas de $C$. flavipes de $24 \mathrm{~h}$ (una hembra del parasitoide por cada larva del hospedero). Una vez expuestas, se realizaron las disecciones diarias de cinco larvas de $D$. saccharalis, previamente colocadas en alcohol etílico (70\%) durante 15 segundos y posteriormente trasladadas a cápsulas Petri que contenían solución salina. En la disección, se realizó un corte lateral entre el penúltimo y el último segmento de la larva, lo facilitó la expulsión de los huevos y larvas de $C$. flavipes por presión de la hemolinfa tal como lo describe Hernández (2010).

Las primeras dos observaciones se realizaron cada 10 horas después de la exposición y posteriormente cada 24 horas. A huevos, larvas y pupas se midió la longitud y el ancho (mm). Las mediciones de huevo se realizaron directo en la solución salina, mientras que las larvas fueron montadas en un portaobjetos usando Hoyer como medio de montaje (Anderson, 1954) para realizar las mediciones. A la par se estimó la duración de cada una de las fases, así como los estadíos que ocurren en la larva.

Todas las observaciones fueron realizadas empleando un estereomicroscopio Leica ${ }^{\circledR}$ S8 Apo con lentes apocromáticos, Microsystems, Wetzlar, Germany, con una magnificación de $10 \mathrm{X}$ a $120 \mathrm{X}$, que tenía incorporado un lente con micrómetro con el que se realizaron las mediciones.

Proporción de sexos. En los Hymenoptera, el tipo de reproducción es la haplodiploidia (van den Bosh et al., 1982), pero ésta a su vez está dividida en 1. Arreonotoquia o mayor proporción de hembras, las cuales provienen de huevos fertilizados y machos provinientes de hembras vírgenes, 2. Deuteriotoquia donde hembras vírgenes dan origen a hembras pero también se producen algunos machos y 3. Telitoquia, donde de hembras vírgenes solo se generan hembras en la progenie. Basado en esto, para determinar la proporción de sexos y el tipo de reproducción, 25 larvas de $D$. saccharalis fueron expuestas a hembras vírgenes de $C$. flavipes y 25 a hembras apareadas de parasitoide. Una vez emergidos los adultos en cada larva se contaron machos y hembras resultantes en la progenie.

Cantidad de larvas parasitadas por una hembra de C. flavipes. A hembras apareadas de $C$. flavipes le fueron 
expuestas una, cinco y siete larvas $D$. saccharalis de 18 días de edad para determinar la cantidad máxima de larvas del hospedero que una hembra puede parasitar. Cada hembra de $C$. flavipes fue colocada en un envase de vidrio de 250 $\mathrm{ml}$ de capacidad cerrado con tela de organdí que se sujetaba al frasco con una banda elástica de PVC. A la tela de organdí se le realizó un orificio de aproximadamente $0,5 \mathrm{~cm}$ de diámetro, por el cual se le ofrecía las larvas al parasitoide. Una vez aguijoneada, cada larva era colocada en un envase plástico de $6 \times 2 \mathrm{~cm}$ (diámetro $\times$ alto) que contenían maíz tierno. A la tapa de este último envase se le hicieron 50 perforaciones equidistantes de $5 \mathrm{~mm}$ de diámetro para permitir la aireación. Este experimento fue repetido diez veces para cada una de las cantidades de larvas señaladas.

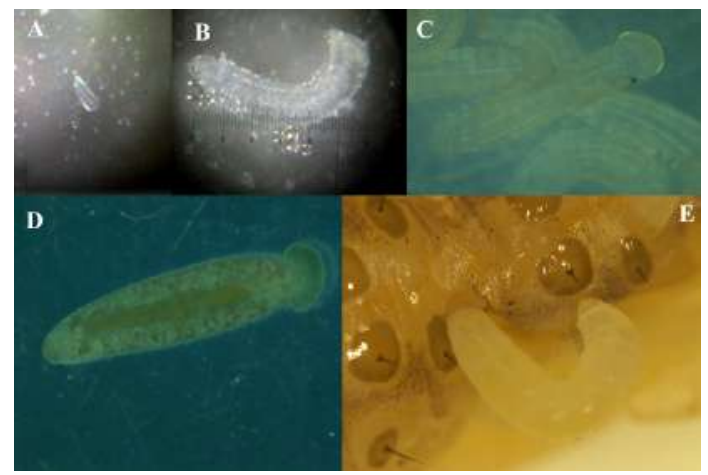

Figura 1. Fases del desarrollo de Cotesia flavipes Cameron. A: Huevo, B: primer estadío larval, C: segundo estadío larval, D: tercer estadío larval.

Medios de cría. Se evaluaron varios medios de crías que incluían dos condiciones de aireación del recipiente de cría (tapa sin perforar y perforada), dos dietas (dieta artificial y natural a base de 10 g de maíz tierno) y dos edades del hospedero (16 y 18 días) que son las edades del hospedero usadas en laboratorios de cría. La dieta utilizada fue modificada de Hensley y Hammond (1968) y fue elaborada en el Laboratorio de Entomología de la Sociedad Agrícola e Industrial San Carlos S.A (Tabla 1). Una de las modificaciones de la dieta fue la sustitución del formaldehido por los ácidos benzoico y sórbico. El formaldehido es usado como anticontaminante en dieta de insectos.

Tabla 1.

Dieta preparada para la alimentación de Diatraea saccharalis modificada de Hensley y Hammond (1968).

\begin{tabular}{ll}
\hline Ingrediente & Cantidad \\
\hline Harina de maíz & $140 \mathrm{~g}$ \\
Levadura de cerveza & $36 \mathrm{~g}$ \\
Germen de trigo & $35 \mathrm{~g}$ \\
Bagazo de la caña & $24 \mathrm{~g}$ \\
Sales de Wesson & $4,6 \mathrm{~g}$ \\
Ácido ascórbico & $5 \mathrm{~g}$ \\
Acido benzoico & $1,4 \mathrm{~g}$ \\
Ácido sórbico & $1 \mathrm{~g}$ \\
Metil parabeno & $1 \mathrm{~g}$ \\
Ampicilina & $750 \mathrm{mg}$ \\
Solución de vitaminas & $30 \mathrm{cc}$ \\
Agua & $500 \mathrm{cc}$ \\
Agar & $12,6 \mathrm{~g}$ \\
\hline
\end{tabular}

Este experimento fue repetido tres veces, usando 50 larvas por tratamiento por repetición para un total de 1200 hospederos evaluados. Los recipientes usados para la cría tenían una dimensión de $5,5 \mathrm{~cm} \times 2 \mathrm{~cm}$ (diámetro $\mathrm{x}$ ancho) y aquellos con aireación contenían 50 perforaciones en la tapa. Para el parasitismo, a cada larva se le colocó una hembra del parasitoide de 24 horas como adulta y previamente apareada siguiendo el mismo procedimiento que fue previamente para la evaluación de cantidad de larvas parasitadas por una hembra de $C$. flavipes. Para este caso se estimó el número de pupas por masa. 
Análisis estadístico. Para las variables de duración de ciclo biológico fueron calculadas las medias para cada una de las fases del desarrollo. Para evaluar los medios de cría, el análisis fue realizado en un arreglo factorial (dos dietas $\mathrm{x}$ dos tipos de recipientes $\mathrm{x}$ dos edades de larva del hospedero), analizando el número de pupas mediante un GLM (Modelo Lineal General, siglas en inglés), y las comparaciones de medias elaboradas para las tres variables clasificatorias por separado y su interacción mediante la prueba de Mínimos Cuadrados $(P<0,05)$.

\section{Resultados y discusión}

\section{Duración del ciclo biológico}

Huevo. Los huevos de C. flavipes mostraron un corion liso y transparente, son alargados con medidas de $0,163 \mathrm{~mm}$ x $0,035 \mathrm{~mm}$ (largo x ancho) (Tabla 2). Además son redondeados hacia los extremos, siendo uno más angosto que el otro (Figura 1A). Estas características han sido descritas, en investigaciones previas (Fajardo y Mendoza, 1993; Astola y Narrea, 2019). Esta fase duró en promedio 2,4 días (Tabla 2) lo que es similar a lo obtenido por Astola y Narrea (2019) e inferior a los 3,2 días reportados por Hernández (2010).

Tabla 2.

Duración (días) y dimensiones (largo y ancho) observadas para las diferentes fases de Cotesia flavipes Cameron.

\begin{tabular}{lccc}
\hline \multicolumn{1}{c}{ Fase } & $\begin{array}{c}\text { Largo } \\
\mathbf{( m m )}\end{array}$ & $\begin{array}{c}\text { Ancho } \\
\mathbf{( m m )}\end{array}$ & $\begin{array}{c}\text { Duración } \\
\text { (días) }\end{array}$ \\
\hline Huevo & 0,163 & 0,035 & $2,4 \pm 0,5$ \\
Larva estadío 1 & 0,748 & 0,175 & $3,5 \pm 0,5$ \\
Larva estadío 2 & 2,850 & 0,780 & $2,5 \pm 0,5$ \\
Larva estadío 3 & 4,367 & 1,014 & $2,6 \pm 0,8$ \\
Pupa & 4,170 & 0,945 & $4,9 \pm 1,2$ \\
\hline
\end{tabular}

Larva. Las dimensiones obtenidas indicaron que la larva transcurre por tres estadíos larvales (Tabla 2; Figura 1). En el primer estadío, la larva es traslúcida con medidas de $0,748 \times 0,175 \mathrm{~mm}$ (largo $\mathrm{x}$ ancho), la cual presentó una vesícula anal pequeña (Figura 1B). Las larvas mudaron al segundo estadío, 3,5 días después de la eclosión de los huevos. Además de esta especie, la presencia de la vesícula anal ha sido observada en otros himenópteros parasíticos (Clausen 1940; Xu et al., 2007; Fischer et al., 2008) y se sugiere que favorece el desarrollo del parasitoide como una adaptación al ambiente interno del hospedero (Pinheiro et al., 2010). Las dimensiones observadas son superiores a las referidas por Fajardo y Mendoza (1993) en un estudio de biología realizado en similares condiciones de temperatura y humedad relativa en Ecuador hace casi tres décadas e inferior a la longitud obtenida por Hernández (2010). La duración del primer estadío resultó mucho más corta (casi la mitad del tiempo) que la reportada por Hernández (2010).

En el segundo estadío, la larva es de color blanquecino con una longitud y un ancho promedio de 2,85 y $0,78 \mathrm{~mm}$, respectivamente; la vesícula anal se observó bien desarrollada, con un sistema digestivo conspicuo, ocupando gran parte del hemocelo (Figura 1C). Este estadío duró 2,5 días. Las dimensiones mostradas en la Tabla 3 son semejantes a las obtenidas por Fajardo y Mendoza (1993) $\mathrm{y}$ superiores a las indicadas por Hernández (2010). La duración del estadío fue similar a la observada por Hernández (2010). 
La larva de tercer estadío midió 4,367 x 1,014 mm; observándose una coloración blanco cremosa, y presentó una vesícula anal bien desarrollada (Figura 1D) a inicio de la muda, pero cuando avanzó en desarrollo a prepupa fue disminuyendo de tamaño. Pinheiro et al. (2010) señalaron que la vesícula anal aumenta de tamaño en los primeros días y luego decrece hacia el final del período larval, cambios de tamaño han sido correlacionados con la actividad de alimentación de la larva del parasitoide. Este estadío se completó en 2,6 días. Las dimensiones de la larva aquí estimadas, superan las reportadas en otras investigaciones (Fajardo y Mendoza, 1993; Hernández 2010). Por otro lado, la duración de este estadío fue 2,5 veces superior a lo referido por Hernández (2010).

El desarrollo de larva transcurrió en unos 8,7 días, lo que fue semejante a lo encontrado en otras investigaciones (Fajardo y Mendoza, 1993; Eduardo et al., 2017), pero superó los siete días observados por Astola y Narrea (2019) y fue inferior a los 9,5 días obtenidos por Hernández (2010). La duración de la pupa resultó inferior a lo detectado en varias investigaciones (Fajardo y Mendoza, 1993; Hernandez 2010; Astola y Narrea 2019).

El ciclo (huevo - emergencia del adulto) duró de 16 días, siendo más corto que los 18,5 días reportados en el estudio previo realizado en el país (Fajardo y Mendoza, 1993). Asimismo, la duración del ciclo fue inferior a la detectada en otras investigaciones (Jiang et al., 2004; Hernandez 2010) y semejante a lo reportado por Astola y Narrea (2019).
Lo señalado anteriormente denota las variaciones en las duraciones de fases y del ciclo total, en las investigaciones realizadas sobre la biología de $C$. flavipes. Dichas variaciones podrían estar asociadas a factores extrínsecos, tales como, condiciones ambientales, alimentación, medios de cría, edad y calidad del hospedero, entre otros, así como, factores intrínsecos de la especie o por la interacción de ambos, que inciden en la actividades biológicas del parasitoide (Khan et al., 2017). Respecto a los factores intrínsecos, la diversidad genética implica variaciones en los aspectos biológicos y ecológicos del parasitoide (Muirhead et al., 2012). En este contexto, $C$. flavipes ha sido reubicada taxonómicamente y denominada como un complejo de cuatro especies, basado en la diversidad genética encontrada que está asociada a sus lugares de origen: C. flavipes, originaria de la región Indo-Asia, Cotesia sesamiae (Cameron) de África, Cotesia chilonis (Matsumara) originaria de China y Japón, así como, Cotesia nonagriae (Oliff) de Australia, Papua y Nueva Guinea (Muirhead et al., 2012). En las regiones geográficas donde se encuentra establecido el parasitoide, C. flavipes, podrían realizarse secuenciamientos de ADN, para corroborar, la existencia de uno o varios miembros que forman este complejo de especies, con el fin de asociar la diversidad genética con los aspectos biológicos.

\section{Proporción de sexos}

Al exponer larvas de $D$. saccharalis a hembras vírgenes de $C$. flavipes solo 
fueron obtenidos machos, mientras que cuando la exposición se realizó a hembras apareadas del parasitoide, en la progenie se obtuvieron aproximadamente dos hembras por cada macho (Tabla 3). Estos resultados muestran que la reproducción de $C$. flavipes es por arrenotoquia corroborando resultados anteriores (Fajardo y Mendoza, 1993; Astola y Narrea, 2019). La proporción de hembras difiere de lo señalado en otros estudios, en los que se obtuvieron, cuatro hembras por cada macho (Fajardo y Mendoza, 1993; Astola y Narrea, 2019).

Tabla 3.

Proporción sexual de Cotesia flavipes Cameron resultante de la progenie de hembras parasitadas y hembras vírgenes.

\begin{tabular}{llclc}
\hline & \multicolumn{2}{c}{$\begin{array}{c}\text { Hembras } \\
\text { apareadas }\end{array}$} & & $\begin{array}{c}\text { Hembras } \\
\text { vírgenes }\end{array}$ \\
${$\cline { 2 - 3 }$} }$ & Individuos & Hembra:Macho & Individuos \\
\hline Hembras & 1142 & $2,11: 1$ & 0 \\
Machos & 542 & & 1075 \\
\hline Totales & $\mathbf{1 6 8 4}$ & & $\mathbf{1 0 7 5}$ \\
\hline
\end{tabular}

\section{Cantidad de larvas parasitadas}

Hembras de $C$. flavipes parasitaron el $100 \%$ de las larvas del hospedero cuando le fue expuesta una sola larva. Igualmente cuando se expusieron cinco y siete larvas del hospedero, en la mayoría de los casos $(87,1 \%)$, la hembra del parasitoide solo parasitó una larva. En las larvas del hospedero parasitadas se formaron masas (grupos de pupas por larva hospedera) que contenían entre 39 a 43 pupas. En el $12,9 \%$ de exposiciones restantes, hembras C. flavipes parasitaron dos larvas del hospedero. De éstas, en un 8,6\% de las dos larvas parasitadas, se formaron dos masas de pupas (una por cada larva del hospedero), una normal (aproximadamente 40) y la otra, pequeña (aproximadamente
16 pupas) y en un $4,3 \%$ de las dos larvas parasitadas se obtuvieron dos masas de pupas pequeñas (por cada larva del hospedero).

Estos resultados coinciden con los obtenidos en investigaciones en diferentes latitudes (Astola y Narrea, 2019; Hernandez, 2010; Manjoo y Bajpai, 2013). Manjoo y Bajpai (2013) señalaron que en un trabajo de laboratorio realizado exponiendo diferentes densidades de larvas del barrenador del tallo, Chilo partellus (Swinhoe) a una hembra de $C$. flavipes, el número de pupas del parasitoide disminuyó cuando se incrementó la densidad de larvas del hospedero.

La capacidad de C. flavipes para parasitar mayoritariamente una larva de su hospedero, probablemente este asociada al hecho que todos los miembros del Complejo C. flavipes son pro-ovigénicos, es decir, las hembras tienen un número de huevos fijos desarrollados al emerger como adulta, cuyas cantidades varían de 150 a 200, lo que dependiendo del miembro del complejo y de las interacciones con los factores extrínsecos puede convertirse en una fecundidad real que oscila entre 30 a 110 huevos (Potting et al., 1997; Muirhead et al., 2010).

\section{Medios de cría}

La Tabla 4 muestra que los tres factores evaluados (dieta, recipiente y edad de la larva hospedera) tuvieron incidencia en el desarrollo de las pupas de C. flavipes. El número de pupas del 
Tabla 4

Número de pupas de Cotesia flavipes desarrolladas según la dieta (natural a base de maíz tierno y dieta artificial), el tipo de recipiente (tapa perforada, tapa no perforada) y la edad de la larva hospedera.

\begin{tabular}{llllll}
\hline Dieta & Pupas & Recipiente & Pupas & Edad larva & Pupas \\
\hline Natural & $46,1 \pm 2,7 \mathrm{a}$ & Perforado & $48,3 \pm \mathrm{a}$ & 18 días & $50,3 \pm 2,0 \mathrm{a}$ \\
Artificial & $42,3 \pm 2,2 \mathrm{~b}$ & No perforado & $40,0 \pm \mathrm{b}$ & 16 días & $38,1 \pm 1,4 \mathrm{~b}$ \\
\hline
\end{tabular}

$R^{2}: 0,86 ; C V: 7,8 ; F=30,22$; Comparaciones de medias realizadas con la prueba de Mínimos Cuadrados $(P<0,05)$.

parasitoide resultó significativamente superior en la dieta a base de maíz tierno, así como en los recipientes con tapa perforada y en las criadas sobre larvas del hospedero de 18 días de edad. Corroborando estos resultados, la Tabla 5 muestra la significancia de la interacción de esos factores, debido a que la mayor cantidad de pupas de $C$. flavipes fue obtenida sobre larvas de $D$. saccharalis de 18 días de edad, alimentadas con maíz tierno y contenidas en recipientes perforados, lo que difirió significativamente del resto de los tratamientos.

El mayor número de pupas se observó en larvas de 18 días de edad, alimentadas con dieta artificial y contenidas en recipientes perforados, sin diferencias con el número de pupas determinadas sobre larvas de 18 días de edad alimentadas con dieta natural o artificial $\mathrm{y}$ criadas tanto en envases perforados como los no perforados (Tabla 5). Los menores números de pupas fueron detectados en larvas del hospedero de 16 días de edad contenidas en recipientes no perforados alimentadas con dieta natural y dieta artificial.

El inferior número de pupas del parasitoide desarrolladas a partir de larvas de 16 días de $D$. saccharalis podría estar asociado al menor tamaño de este hospedero comparado con la larva de 18 días de edad. Clausen (1940) señaló que el tamaño del hospedero está directamente relacionado con el desarrollo de la progenie en parasitoides gregarios $y$ en consecuencia, de hospederos más pequeños se obtienen menor cantidad de parasitoides.

Tabla 5.

Número de pupas de Cotesia flavipes Cameron, por larva de Diatraea saccharalis F. en condiciones de laboratorio.

\begin{tabular}{ll}
\hline Tratamientos & \multicolumn{1}{c}{ Pupas } \\
\hline L16+DN+RP & $42,8 \pm 1,5 \mathrm{~cd}$ \\
L16+DA+RP & $41,7 \pm 1,8 \mathrm{~cd}$ \\
L16+DN+RNP & $37,8 \pm 0,7 \mathrm{~d}$ \\
L16+DA+RNP & $30,7 \pm 1,7 \mathrm{e}$ \\
L18+DN+RP & $57,8 \pm 3,8 \mathrm{a}$ \\
L18+DA+RP & $51,2 \pm 2,3 \mathrm{~b}$ \\
L18+DN+RNP & $46,7 \pm 1,5 \mathrm{bc}$ \\
L18+DA+RNP & $45,5 \pm 1,5 \mathrm{bc}$ \\
\hline
\end{tabular}

L16: larva de 16 días; L18 = larva de 18 días; $\mathrm{DN}=$ Dieta natural, DA $=$ Dieta artificial; $\mathrm{RP}=$ recipiente perforado; RNP = recipiente no perforado. $R^{2}: 0,86$; $C V: 7,8 ; \quad F=30,22$; Comparaciones de medias realizadas con la prueba de Mínimos Cuadrados $(P<0,05)$.

La aireación del recipiente es otro factor de importancia en el medio de cría, debido a que evita que se acumule la humedad que favorece el desarrollo de hongos contaminantes. Por otro lado, la disponibilidad de oxígeno parece influir con el tamaño que desarrolla el cuerpo de un insecto en cámaras de cría. Callier y Nijhout (2011) realizaron trabajos de 
laboratorio suministrando oxígeno a larvas del lepidóptero, Manduca sexta L. y demostraron que los niveles de oxígeno influyeron en el tamaño del cuerpo en los insectos evaluados concluyendo así que la hipoxia redujo el tamaño corporal.

La dieta artificial posiblemente resultó menos adecuada para la cría porque se degradaba más rápido debido a la contaminación que fue observada durante este estudio, y por tanto los compuestos sustitutos del formaldehido usados en la misma no parecieron tener efecto. Tal como se refirió anteriormente, el formaldehído es un compuesto químico, ampliamente utilizado como anticontaminante en la composición de dietas artificiales para la cría de insectos, pero desafortunadamente, quienes usan este compuesto para crías de insectos, pueden estar expuestos a niveles altos del producto que podría conducir a envenenamientos y problemas de salud, tales como, alergias, asma, lesión pulmonar y cáncer (Eduardo et al., 2017).

Se hace necesario evaluar en las dietas otros componentes anticontaminantes para sustituir o al menos disminuir las cantidades de formaldehído. De hecho, están siendo adelantadas investigaciones con resultados que refieren una influencia positiva del 2-fenilfenol como componente anti-contaminante de la dieta que aparentemente no causan efectos adversos sobre el desarrollo del parasitoide (Eduardo et al., 2017).

Estos resultados aportan elementos a ser considerados en la cría masiva de $C$. flavipes. Larvas de 18 días de edad del hospedero, así como, los recipientes perforados, podrían ser utilizadas como parte de los medios de cría. Por otro lado, es necesario seguir evaluando sustitutos del formaldehido como anticontaminante para incrementar la calidad de la dieta de las larvas hospederas.

\section{Agradecimientos}

Al proyecto titulado: "Identificación de las principales plagas, enemigos naturales y virosis en algunos cultivos de importancia en Ecuador y Venezuela" por el soporte académico dado a esta investigación.

\section{Referencias}

Anderson, L.E. (1954). Hoyer's solutions as a rapid permanent mounting medium for bryophytes. The Bryologist, 57, 242-244.

Astola, S. y Narrea, M. (2019). Biología y comportamiento de Cotesia flavipes Cameron (Braconidae) parasitoide de Diatraea saccharalis Fabricius (Crambidae). Ecología Aplicada, 18(1), 77-83.

Aya, V., Echeverri, C., Barrera, G. y Vargas, G. (2017). Cotesia flavipes (Hymenoptera: Braconidae) as a Biological Control Agent of Sugarcane Stem Borers in Colombia's Cauca River Valley. BioOne Research Evolved, 100(4), 826-830.

Callier, V. y Nijhout H. F. (2011). Control of body size by oxygen supply reveals size-dependent and size-independent mechanisms of molting and metamorphosis. Proceedings of the National Academy of Sciences, 108(35), 14664-9.

Clausen, C. P. (1940). Entomophagous insects. New York, USA: McGraw-Hill. Edit. 
Cordoba, L. y Soto, A. (2015). Susceptibility of Diatraea saccharalis (F) (Lepidoptera: Crambidae) to different entomopathogenic fungi in sugarcane. Boletín Científico. Centro de Museos. Museo de Historia Natural, 19(2), 95-103.

Eduardo, W. I., Renato O., Luis V., dos Santos J., dos Santos R. y de Bortoli S. (2017). Alternative to formaldehyde in artificial diet for sugarcane borer and its effects on the parasitoid. SC Sciencie with quality, 8(2), 194-201.

Fajardo, J. y Mendoza, J. (1993). Biología, multiplicación y liberación de Cotesia (=Apanteles) flavipes para el control del barrenador del tallo Diatraea saccharalis en maíz. Comunicación Técnica no. 24. Quevedo, EC: INIAP, Estación Experimental Tropical Pichilingue, Departamento de Entomología.

Ferrer, F., y Liniares, B. A. (1990). Introducción de Cotesia flavipes Cameron (Hymenoptera: Braconidae) para el control de Diatraea spp. (Lepidóptera: Pyralidae) en Venezuela. Probioagro S.A., 8(1), 5-11.

Fischer, M., Tormos, J., Pardo, X. y Asís, J.D. (2008). Description of adults, preimaginal phases, and the venom apparatus of a new species of Aspilota Förster from Spain (Hymenoptera: Braconidae). Zoological Studies, 47(3), 247-257.

Hensley, S. D. y Hammond, A. M. (1968). Laboratory Techniques for Rearing the Sugarcane Borer on an Artificial Diet. Journal of Economic Entomology, 60(1), 1742-1743.

Hernandez, D. (2010). Estudio de algunos aspectos biológicos de Cotesia flavipes Cameron (Hymenoptera: Braconidae) parasitoide de Diatraea saccharalis Fabricius (Lepidoptera: Crambidae). Entomotropica, 25(2), 69-81.

Intituto Nacional de Estadistica y Censos (INEC). (2017). Encuesta de Superficie $y$
Producción Agropecuaria Continua. Recuperado de:

https://www.ecuadorencifras.gob.ec/docu mentos/web-inec/Estadisticas_agropecua rias/espac/espac-2016/Presentacion\%20E SPAC\%202016.pdf.

Jiang, N., Sétamou, M., Ngi-Song, A. J. y Omwega, C. O. (2004). Performance of Cotesia flavipes (Hymenoptera: Braconidae) in parasitizing Chilo partellus (Lepidoptera: Crambidae) as avected by temperature and host stage. Biological Control, 31(2), 155-164.

Khan, J., Ali R., Blouch A., Javed H., Ul Haq E., Mehmood T., y Rasool A. (2017). Biological parameters of Cotesia flavipes reared on different larval instars of Chilo partellus under laboratory conditions. Journal of Entomology and Zoology Studies, 5(3), 829-832.

Manjoo, S., y Bajpai, N. K. (2013) Performance of Cotesia flavipes Cameron (Hymenoptera: Braconidae) parasitizing Chilo partellus (Swinhoe) (Lepidoptera: Crambidae) as affected by size of ovipositional chambers. Journal of Biological Control, 27(1), 35-38.

Mendoza, J., Gualle, D., y Gomez, P. (2009). Manejo de Plagas. Cincae (1 y 2), 19-20.

Muirhead, K. A., Sallam, N. y Austin, A. D. (2010) Life history traits and foraging behaviour of Cotesia nonagriae (Olliff) (Hymenoptera: Braconidae), a newly recognised member of the Cotesia flavipes complex of stemborer parasitoids. Australian Journal of Entomology, 49, 56-65.

Muirhead, K. A., Murphy, N. P, Sallam N, Donnellan, S. C. y Austin AD. (2012). Phylogenetics and genetic diversity of the Cotesia flavipes complex of parasitoid wasps (Hymenoptera: Braconidae), biological control agents of lepidopteran stemborers. Molecular Phylogenetics and Evolution, 63, 904-914. 
Pérez, H. y Rodríguez, I. (2018). Cultivos tropicales de importancia económica en Ecuador (arroz, yuca, caña de azúcar y maiz). Recuperado de:

file:///C:/Users/Windows/Downloads/Cu ltivosTropicalesDeImportanciaEconomic $\mathrm{a} \% 20(1)$.pdf.

Pinheiro, D. O., Rossi, G. D. y Consoli, F. L. (2010). External morphology of Cotesia flavipes (Hymenoptera: Braconidae) during larval development. Zoologia (Curitiba), 27(6), 986-992.

Potting, R. P. J., Overholt, W. A., Danso, F. O. y Takasu, K. (1997). Foraging behavior and life history of the stemborer parasitoid Cotesia flavipes (Hymenoptera: Braconidae). Journal of Insect Behavior, 10, 13-29.

Vacari, A. M., Bortoli, S. A., Borba, D. F. y Martins, M. I. E G. (2012).Quality of Cotesia flavipes (Hymenoptera: Braconidae) reared at different host densities and the estimated cost of its commercial production. Biological Control, 63(2), 102-106.

Van den Bosh, R., Messenger, P. S. y Gutierrez, A. P. (1982). An Introduction to Biological Control. New York and London: Plenum Press.

Xu, P., Wan, Z. W., Chen, X. X., Liu, S. y Feng, M. G. (2007). Immature Morphology and Development of Opius caricivorae (Hymenoptera: Braconidae), an Endoparasitoid of the Leafminer Liriomyza sativae (Diptera: Agromyzidae). Annals of the Entomological Society of America, 100(3), 425-432. 
